\title{
Effect of inorganic nitrate and beetroot juice supplementation on blood pressure: a systematic review
}

\author{
I. Ogbonmwan, M. Siervo, J. Lara, J. C. Mathers \\ Human Nutrition Research Centre, Institute for Ageing and Health, Newcastle University, NE4 5PL, UK
}

Inorganic nitrate and beetroot juice supplementation have both been associated with a decrease in blood pressure in humans ${ }^{(1)}$. The effects seem to be mediated by an increased non-enzymatic conversion of nitrate into nitric oxide, a key endothelial-derived vasodilator ${ }^{(2)}$.

The aim of the study was to perform a systematic review of randomized clinical trials to evaluate the effects of inorganic nitrate and beetroot supplementation on systolic (SBP) and diastolic (DBP) blood pressure in humans.

We searched the Embase, Medline and Scopus medical databases from inception until March 2012. The databases were searched for title and abstract and were restricted to human, randomized, placebo controlled clinical trials published in English. Predefined search terms (nitrate, blood pressure, hypertension, vascular, nitrate, nitric oxide, beet root) and Boolean operators (AND, OR, “", *) were used to increase the power of the search strategy. The protocol for the study has been registered with PROSPERO International prospective register of systematic reviews (CRD42012002144). We identified 8284 records and 3135 articles were included in the database after removal of duplicates. Titles and abstracts of each article were independently evaluated for eligibility by two members of the research team (IO, MS) and discrepancies were resolved by discussion with a third reviewer (JL). Twenty-two articles were selected and the full text of each manuscript was obtained for data extraction. The reference lists of the selected articles and relevant reviews were searched to retrieve other potential eligible articles. Eight articles were excluded after data extraction and 14 studies were included in the database for the final analysis (Table 1).

Table 1. Effects of beetroot and inorganic nitrate supplementation on systolic (SBP) and diastolic (DBP) blood pressure in randomized clinical trials

\begin{tabular}{|c|c|c|c|c|c|}
\hline \multicolumn{3}{|c|}{ Beetroot Juice Supplementation } & \multicolumn{3}{|c|}{ Inorganic Nitrate Supplementation } \\
\hline Author & $S B P$ & $D B P$ & Author & $S B P$ & $D B P$ \\
\hline (3) Webb et al. (2008) & $\downarrow$ & - & (11) Larsen et al. (2006) & - & $\downarrow$ \\
\hline (4) Bailey et al. (2009) & $\downarrow$ & - & (12) Larsen et al. (2007) & $\downarrow$ & $\downarrow$ \\
\hline (5) Bailey et al. (2010) & $\downarrow$ & $\downarrow$ & ${ }^{(6)}$ Kapil et al. $(2010)^{*}$ & $\downarrow$ & $\downarrow$ \\
\hline (6) Kapil et al. (2010)* & $\downarrow$ & - & (13) Larsen at al. (2010) & - & - \\
\hline (7) Vanhatalo et al. (2010) & $\downarrow$ & $\downarrow$ & (14) Lidder et al. (2011) & $\downarrow$ & $\mathrm{n} / \mathrm{a}$ \\
\hline (8) Gilchrist et al. (2011) & - & - & (15) Bahra et al. (2012) & $\downarrow$ & - \\
\hline (9) Lansley et al. (2011) & $\downarrow$ & - & (16) Cermak et al. (2012 & - & - \\
\hline${ }^{(10)}$ Lansley et al. (2011) & $\downarrow$ & - & & & \\
\hline
\end{tabular}

$\downarrow=$ Significant decrease in BP; - = No significant change in BP; n/a $=$ BP values were not available; $*=$ results reported in the same article.

All studies were characterized by a crossover, double-blind, placebo controlled, randomized study design. Thirteen studies included healthy volunteers and one study was conducted in patients with type 2 diabetes. Large heterogeneity was observed in the duration of the interventions (range: 3 hours - 15 days) but it was not associated with changes in SBP and DBP. An improvement in SBP was observed in 11 studies whereas only 5 studies reported a decrease in DBP. Beetroot juice supplementation did not have an effect on BP in type 2 diabetic patients.

Inorganic nitrate and beetroot juice supplementation are both associated with beneficial effects on vascular homeostasis, which could be mechanistically explained by an increased generation of nitric oxide. The majority of the studies have been conducted in healthy individuals and therefore the benefits of inorganic nitrate and beetroot supplementation in subjects at high risk of cardiovascular diseases remain to be established.

1. Gilchrist M et al. (2011) Cardiovasc Res 89(3): 492-498.

2. Lundberg J et al. (2008) Nat Rev Drug Discov 7(2): 156-167.

3. Webb A et al. (2008) Hypertension 51(3): 784-790.

4. Bailey S et al. (2009) J Appl Physiol 107(4): 1144-1155.

5. Bailey S et al. (2010) J Appl Physiol 109(1): 135-148.

6. Kapil V et al. (2010) Hypertension 56(2): 274-281.

7. Vanhatalo A et al. (2010) Am J Physiol 299(4): R1121-31.

8. Gilchrist M et al. (2011) J Hum Hyper 25(10): 626.

9. Lansely K et al. (2011) Med Sci Sports Exerc 43(6): 1125-31.

10. Lansely K et al. (2011) J Appl Physiol 110(3): 591-600.

11. Larsen F et al. (2006) N Engl J Med 355(26): 2792-2793.

12. Larsen F et al. (2007) Acta Physiol (Oxf) 191(1): 59-66.

13. Larsen F et al. (2010) Free Radic Biol Med 48(2): 342-347.

14. Lidder S et al. (2011) Nitr Ox-Biol \& Chem 24: S32.

15. Bahra $\mathrm{M}$ et al. (2012) Nitr Ox Article In Press.

16. Cermak N et al. (2012) Int J Spt Nut \& Ex Met 22(1): 64-72. 